Stanisław Lewiński, Karol Zaremski

\title{
EXAMPLES OF OBJECT-ORIENTED CLASSIFICATION PERFORMED ON HIGH-RESOLUTION SATELLITE IMAGES
}

\begin{abstract}
Information about the types of land cover and its use is obtained by the visual interpretation of the color composite of satellite images or by the use of automatic classification algorithms. For obvious reasons, the automatic classification methods make it possible to obtain information quicker and much faster than the traditional interpretation method.

The commonly used automatic methods of satellite image classification, based on supervised or unsupervised classification algorithms, are the most accurate when used with low resolution images. In the case of images with 1-meter-sized pixels, showing a diversity of land cover forms, it is not possible to obtain satisfactory results.

New classification techniques, based on object-oriented classification algorithms, have been developing for a couple of years now. In contrast to the traditional methods, the new operating procedure does not involve the classification of single pixels, but of entire objects, into which the content of the satellite image is divided. Aside from the spectral values of the pixels, the shape of the objects created by the pixels and the relationships between the objects, are also considered during the analysis. Similar to visual interpretation, variation in the texture of the image can also be taken into account in this case.

The aim of this article is to present the possibility of using high density satellite images in object-oriented classification. The classification presented is that of a high-rise built area in Wrocław and of bridges on the Vistula River in Warsaw.
\end{abstract}

Key words: object-oriented classification, land use, satellite images, eCognition

\section{OBJECT-ORIENTED CLASSIFICATION}

Methods of multispectral satellite image classification, founded on supervised and unsupervised classification algorithms, are based on the so-called pixel method of analysis and image classification. First, following a procedure called "training," statistical parameters describing each of the classes are specified. Then, the spectral values of each of the pixels of the image are analyzed. The pixel qualifies into one of the classes, if it fulfills the requirements specified by the statistical parameters.

The presumptions of traditional classification methods most often prove true in the case of low resolution satellite images, e.g. LANDSAT MSS. On the other hand, when using new generation images, characterized by a higher spatial and spectral resolution, it is difficult to obtain results which are fully 
satisfactory. A good example here might be the CORINE program, as part of which a European land cover and land use database is being created, based on LANDSAT TM images (Corine, 1993). The entire image interpretation process is being done visually, since automatic classification methods are not able to produce good results. In the case of the new-generation, 1-meter-resolution satellite images, such as IKONOS or QuickBird, classification is an even more elaborate task.

The development and application of new classification techniques is currently a highly important research area and application issue. There exists an actual need for the creation of tools, which can automatically supply the data obtained from satellite images, to the geographic information systems.

Object-oriented classification is a new classification method. It is utilized by the eCognition software (eCognition, 2001), in which new image-analysis tools are introduced. Here, in contrast to methods used until now, groups of pixels called objects, not the individual pixels of the image themselves are examined during the classification process.

Objects are created during the segmentation process, when the image is subdivided into groups of pixels which have a similar local contrast value. The scale factor, which determines the size of the objects, is the basic parameter in their creation. The elements analyzed are not only the spectral values of an object, but also its shape, texture and the course of its boundary with other, neighboring objects. By applying the correct scale factor, it is possible to obtain objects with boundaries the paths of which closely resemble those in the visual interpretation of the satellite image. It is acceptable that the segmentation of the image takes place in multiple phases. Based on the already existing objects, new, higher-order (larger) or lower-order (smaller) objects are created. The content of the image is represented by the object layers.

The eCognition software's classification process is based upon the fuzzy set theory, used in the "nearest neighbor" method of classification and in the membership function (Brandt, Mather, 2001). By following these principles, it is possible to classify objects, using information about each individual object and also about the relations existing between the objects. Besides analyzing spectral reflection information, one can analyze the shape of the object and its ties to its surroundings, which are composed of adjacent objects or objects of higher- or lower-order. As an example: the texture of an object is calculated based on the spectral values of the objects present at the lower level.

A highly important feature of the software is the ability it gives the user for building a hierarchical class structure. Each class is delimited by a set of rules associated with the "nearest neighbor" algorithm and by the membership functions. A characteristic feature of the class structure is the inheritance of definitions and the creation of groups of classes with common semantic meanings (e.g. the class "low-density built area" is not spectrally homogeneous, but is composed of classes representing buildings, their shadows and the space between them). 
The actual classification process is carried out relatively quickly, because the elements being analyzed are whole objects, not individual pixels. The classification process can be carried out following a single calculation, or a cycle of calculations, in which the number of reiterations is dependent upon the number of relationships existing between the objects. The algorithm was devised mainly with the classification of high resolution satellite images, as well as radar and aerial images, in mind (Bauer, Steinnocher, 2001). It can also be successfully applied in the classification of medium- and low-resolution images (de Kok et al., 2002).

\section{THE CLASSIFICATION OF HIGH-RISE BUILT AREAS ON QUICKBIRD IMAGES}

QuickBird images are high-resolution - so-called "new generation" - satellite images. The image of the Earth's surface is recorded in four spectral bands: $0.45-0.52 \mu \mathrm{m}$ (blue), $0.52-0.60 \mu \mathrm{m}$ (green), $0.63-0.69 \mu \mathrm{m}$ (red) and $0.76-0.90 \mu \mathrm{m}$ (near-IR), by a multispectral scanner with a spatial resolution of $2.8 \mathrm{~m}$. Simultaneously, a panchromatic image within the range of $0.45-$ $0.90 \mu \mathrm{m}$ and with the spatial resolution of $0.7 \mathrm{~m}$, is recorded. Here, the classification was done based on the example of a fragment of an image, which was recorded on April 26, 2003 (and made available by Geosystems Polska). The color composite is presented in Fig. 1.

The QuickBird image shows the Kozanów residential complex in Wrocław. Individual high-rise buildings and multiple section apartment buildings create a pattern typical for residential complexes. Between the tall (10 story) buildings, there are expansive green areas (grass lawns), playgrounds, parking lots, transportation routes and individual, low-rise buildings. The complex borders forested terrain (a forest park) to the south and a complex of single-family houses (a low-rise built area) to the east, while its northern boundary is formed by an expressway, behind which are shopping and resort/recreation areas, situated around a water reservoir.

The analysis of the satellite image was begun by conducting a traditional supervised classification, using the minimum distance algorithm. In the classification process, four multispectral bands and one panchromatic band was used. Based on the analysis of spectral reflections, it was decided to delimit six classes: built areas, forest areas, green areas, bare soil areas (without vegetation), transportation areas and water. The result of the classification is presented in Fig. 2.

In comparing Figs. 2 and 1, one can notice that while most of the satellite image was classified correctly, there are highly visible errors, mostly in association with the class "built areas" and its surroundings. Certain transportation infrastructure surfaces had been classified as built, since asphalt surfaces are spectrally similar to the bituminoid materials used for roofing surfaces on buildings. Another error, typically committed in classification, is 


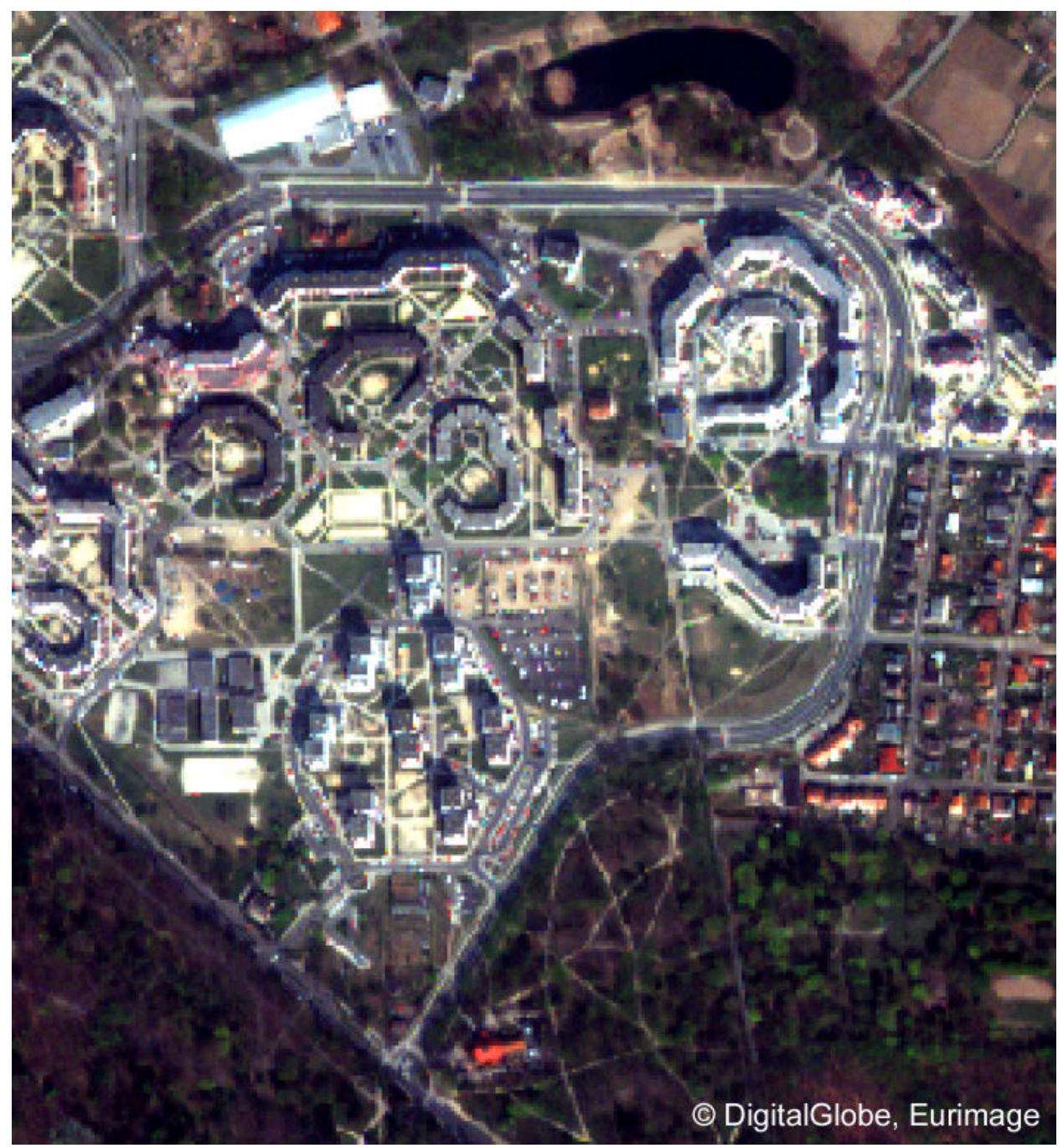

Fig. 1. The color composite of the QuickBird satellite image, Wrocław, Kozanów residential complex.

the inclusion of image pixels associated with the shoreline of the water reservoir and with the linear structures at the borders of wooded areas which are actually transportation routes (walkways) in the forest - within the class "built areas." Due to similar spectral reflection values, the shadows of tall buildings, visible in the image, were repeatedly classified as water. At the borders of wooded areas, there also are single pixels which had been erroneously classified as water. Following the visual analysis, one can determine that the supervised classification method, founded solely on the 


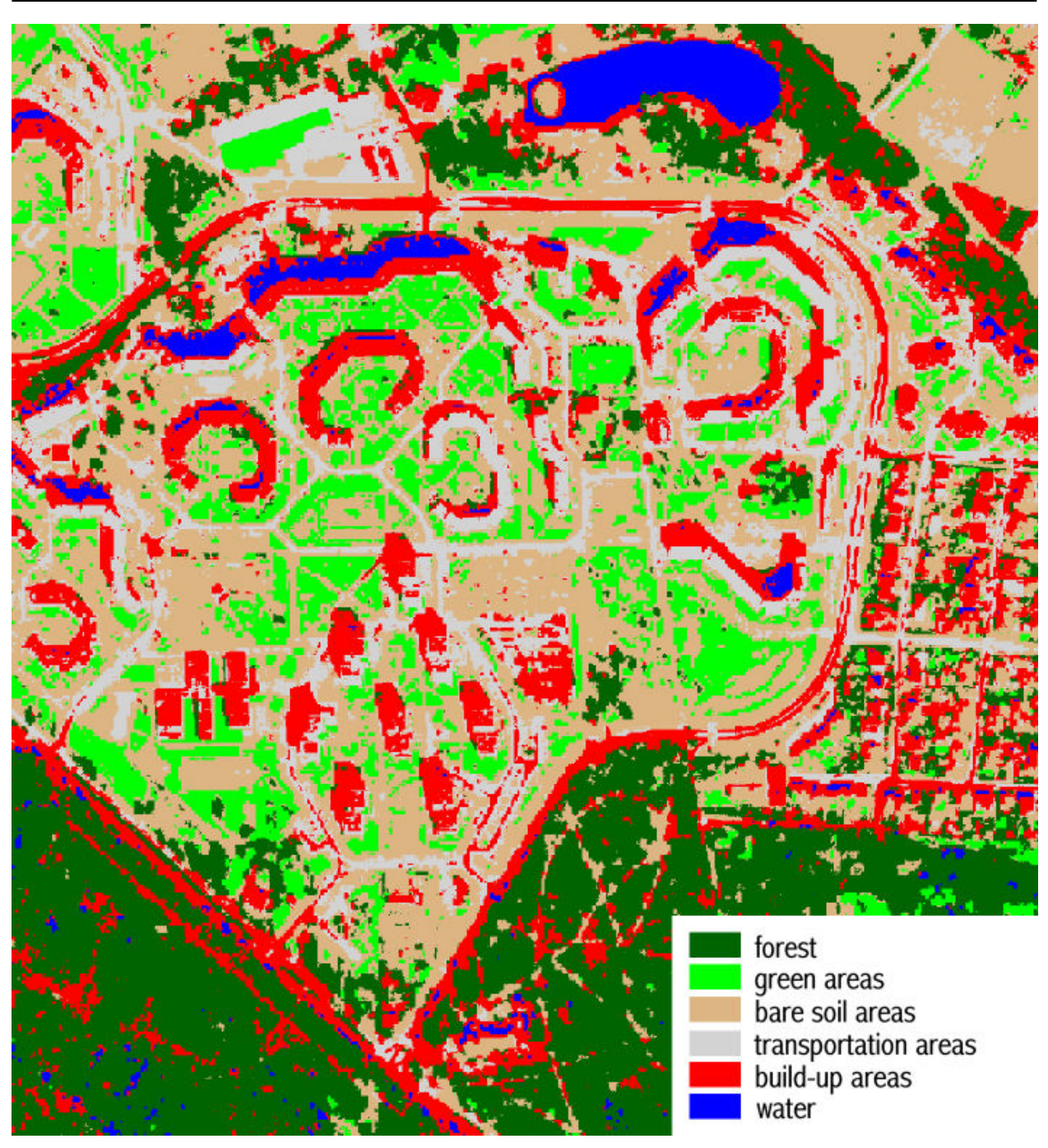

Fig. 2. Supervised classification of the QuickBird image.

pixel values of the satellite image, does not delimit the class "built areas" with what could be called reasonable accuracy.

Basing on the same satellite image, object-oriented classification was carried out in order to mark out only the tall buildings. Classifying them by the traditional methods is a task that is virtually impossible to carry out. Object-oriented classification was begun by the segmentation of the image. The content of the image was subdivided into objects, or groups of pixels. The scale factor, determining the size of the objects, was chosen in such a way that the borders of the delineated areas would correspond with the feature 


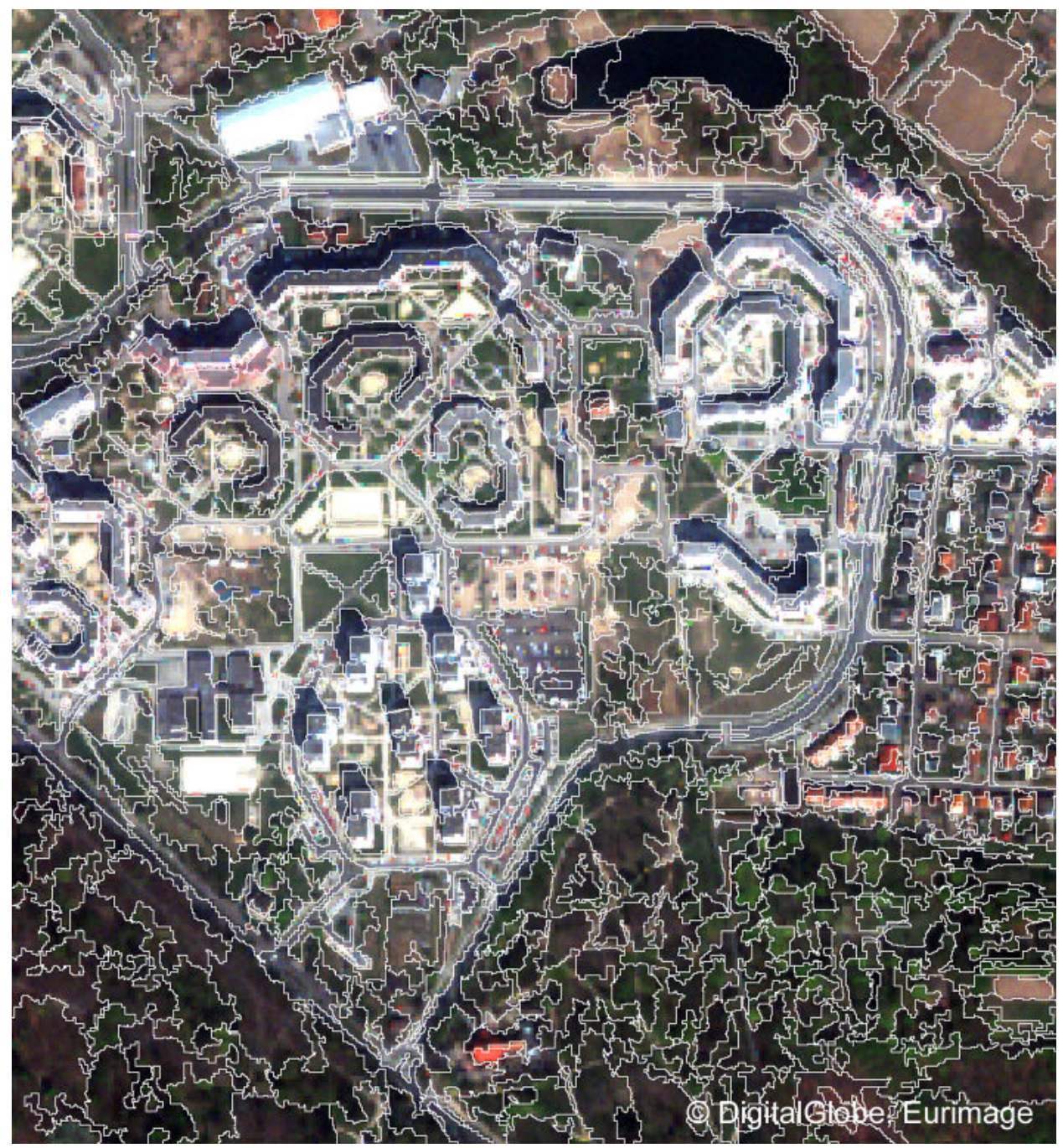

Fig. 3. Object borders following segmentation on the QuickBird image.

patterns (classes of land cover) visible in the image. In Fig. 3, the object boundaries used during the process of classification are presented with the satellite image in the background.

Only two classes were identified: "high-rise built area" and "shadows," as well as the following relation occurring between them: next to an object of the class "high-rise built area" there must always be an object of the class "shadow." The division of objects into two classes was carried out in two stages: first, based on the spectral values of the pixels constituting the objects (taking into account the multispectral bands and the panchromatic band), and then by applying the defined relations between the classes. 


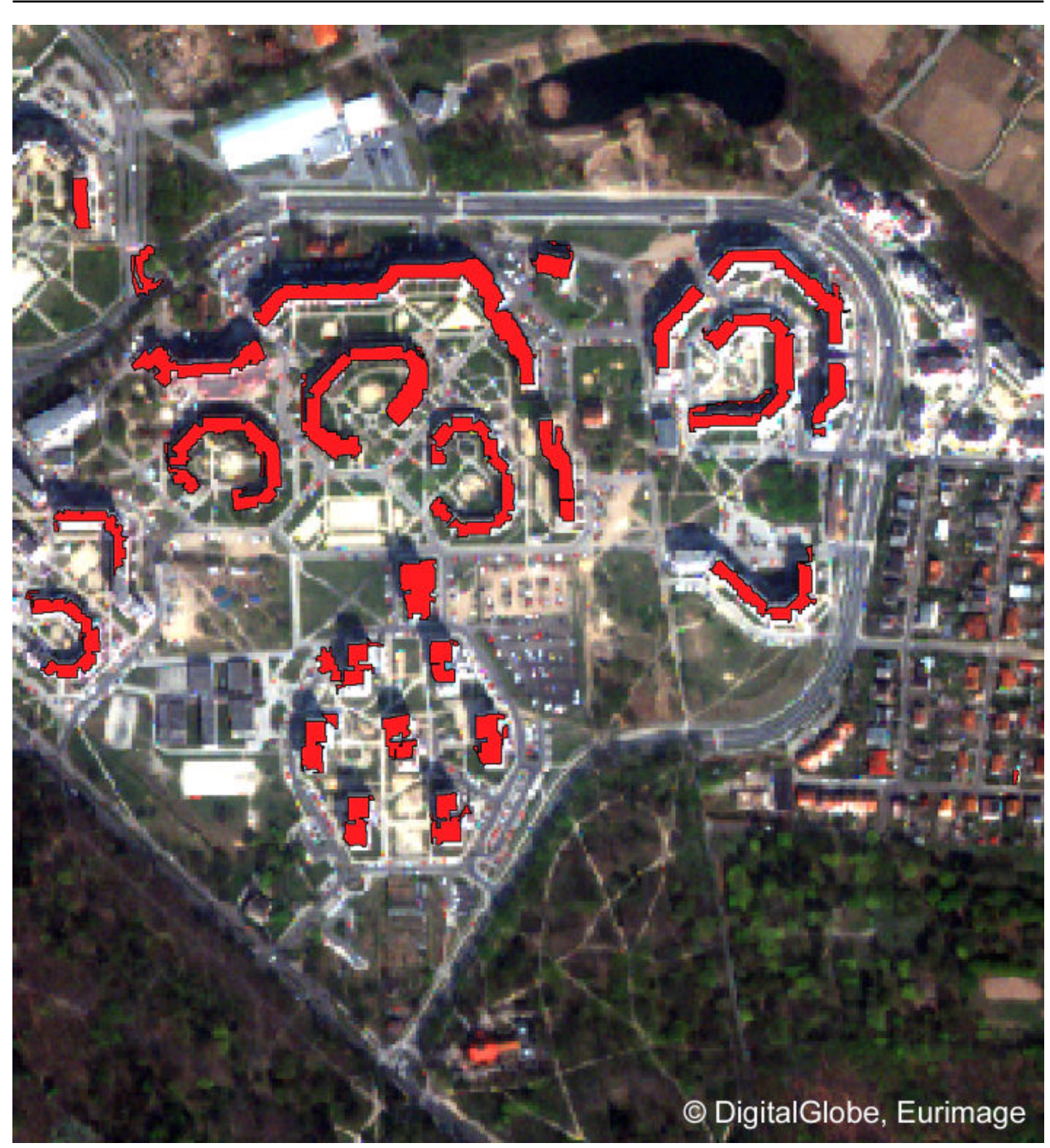

Fig. 4. Object-oriented classification of the high-rise built area on the QuickBird image.

The result of the object-oriented classification process in the case of tall buildings (red color) is presented in Fig. 4, with the color layout of the QuickBird image in the background. All of the tall apartment buildings within the residential complex had been identified correctly. Errors in classification are visible in the upper-left and the top section of the image. A fragment of the road junction and a fragment of the concrete plaza adjoining one building had been misclassified. In addition, sections of two tall buildings had not been classified correctly. Despite the errors mentioned, the outcome of the classification process should be regarded as very good, especially if compared with the classification of the built areas done by the traditional method (Fig. 2). 
THE CLASSIFICATION OF BRIDGES ON THE IKONOS IMAGE

Visually identifying the characteristic shape of a bridge over a river is on a satellite image, a plain and simple task. Carrying out the same task automatically is much more complicated. Using automatic methods of classification, it is not possible to identify the class "bridge," since it is not possible to unambiguously differentiate it from the other anthropogenic classes. The spectral reflection of the bridge is the sum of the spectral reflections of the steel supporting structure, and of the road surface, which is most often bituminous or concrete. These are the only parameters which can be specified for the purpose of correctly identifying a structure that joins the two banks of a river.

By using the IKONOS satellite image - which was recorded on April 29, 2000 and shows the Powiśle district of Warsaw, as well as bridges on the Vistula - as an example, an attempt was made to classify the bridges. (The image comes from the archives of the Laboratory for the Remote Sensing of the Environment, at the Faculty of Geography and Regional Studies.)

The IKONOS satellite image is composed of four multispectral bands: $0.45-0.52 \mu \mathrm{m}, 0.52-0.60 \mu \mathrm{m}, 0.63-0.69 \mu \mathrm{m}$ and $0.76-0.90 \mu \mathrm{m}$, with the spatial resolution of $4 \mathrm{~m}$, as well as with a panchromatic band $0.45-0.90 \mu \mathrm{m}$ with the spatial resolution of $1 \mathrm{~m}$. Similarly as in the case of the high-rise built area, the process of classifying bridges began by segmentation, or the subdividing the content of the image into objects. The segmentation was carried out in two phases. First, a layer of relatively small objects was created, which were used to obtain information about spectral values. Next, a layer of (larger) super-objects was created. The aspect of shape, which should accurately describe linear structures, was given special consideration in the creation of these objects. To enable the identification of the bridges, relations existing between the objects were defined. A "bridge" must be flanked by at least two objects classified as water. Furthermore, its shape must be relatively long and narrow. Not only is a structure spanning across the water seen as a bridge, but so is its continuation in the form of a viaduct.

The result of the classification is presented in Fig. 5, with the color layout of the IKONOS image as the background. The bridges are represented by the three red linear objects. They are (from the north): Most Syreny (The Bridge of the Mermaid), Most Średnicowy (The Cross-town [Rail] Bridge) and Most Poniatowskiego (The Poniatowski Bridge). Right next to Most Syreny, a fourth bridge being constructed is visible - Most Świętokrzyski (The Świętokrzyski Bridge). Yet, it was not classified as a bridge, since at the time when the picture was recorded, its structure was not yet a continuous object joining the two banks of the river. The eastern portion of Most Syreny does not end directly at the water's edge. The object's parameters were intentionally chosen to also mark the section of the river crossing running directly over the low riverbank. The bridge in the middle is the rail bridge - Most Średnicowy. Sections of its structure that were classified as a bridge were 


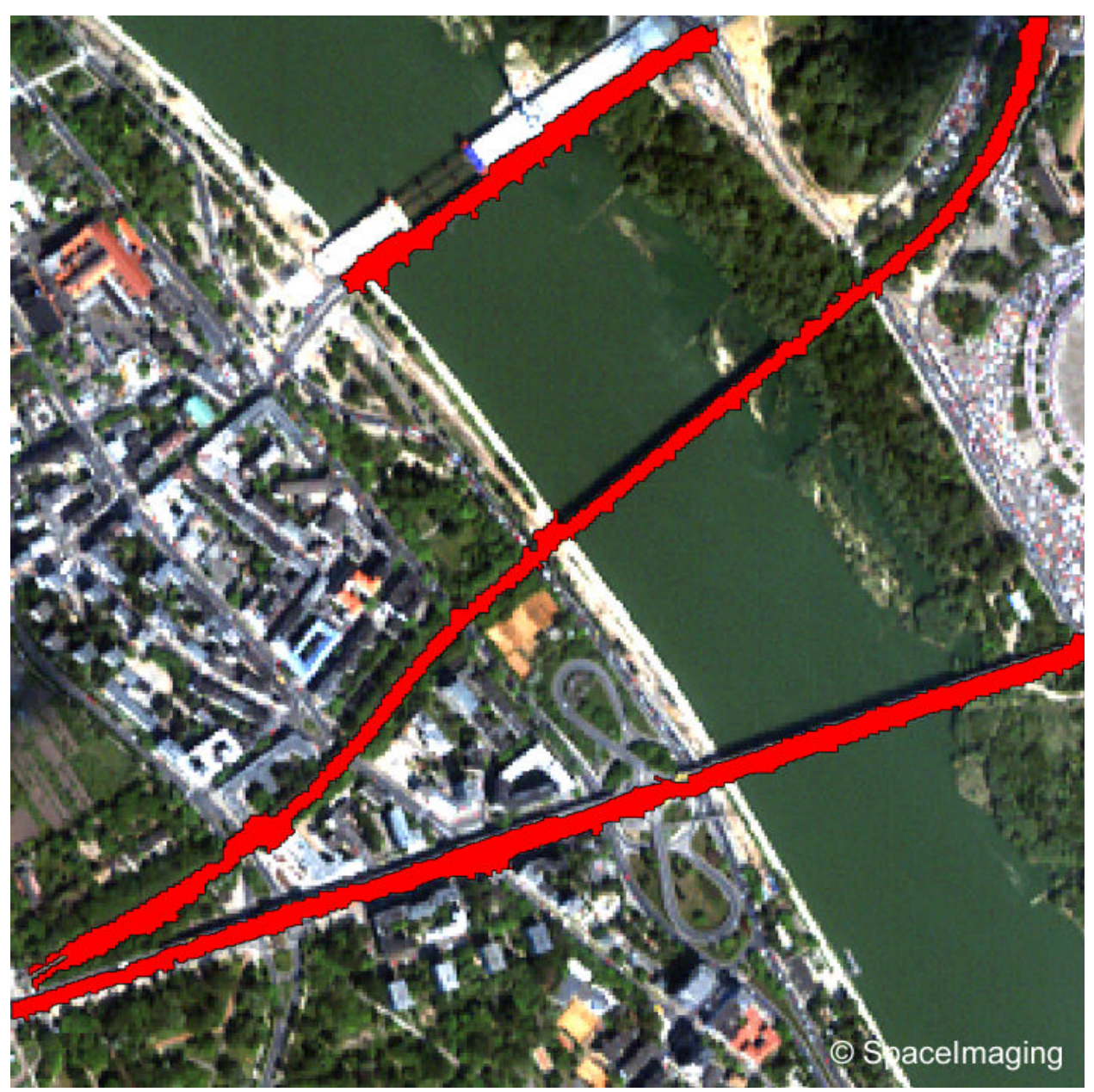

Fig. 5. Object-oriented classification of Warsaw bridges on the IKONOS image.

the section running over the water, as well as the viaducts constituting its extensions: the viaduct running over the Powiśle district on the western bank, and the viaduct leading onto the high embankment on the eastern bank. Most Poniatowskiego is also classified along with a viaduct - the extension running from the Vistula itself to the edge of its high riverbank.

In one section of the image, clearly visible are numerous transportation arteries, which were not classified as bridges or viaducts, even though in their spectral element, they highly resemble the surface of a bridge joining two sides of a river. Performing the classification correctly was possible after correctly identifying the relations existing between the objects generated during the segmentation process. 


\section{REVIEW}

Object-oriented classification provides hitherto unseen possibilities in the area of automatic satellite image interpretation. The emergence of the new classification technique has coincided with the development of the operational usage of multi-resolution satellite images on which the appearance of the Earth's surface can be recorded with accuracy greater than 1 meter. Such high spatial resolution makes it possible to, by visual interpretation, obtain results that approximate those of aerial photographs. At the same time however, the great degree of detail present in the images constitutes a significant impediment in the process of automatic classification.

The classification presented in the examples would have been practically unfeasible, were one to apply the traditional methods of image processing. Object-oriented classification makes it possible not only to determine the class of land cover or land use, but also to recognize the function that is served by the object.

The subdivision of an image's content into objects, to which is tied not only spectral reflection information, but also definitions of the relations existing between the objects, likens automatic classification to the processes of perceptional analysis in visual interpretation.

The data used in classification, structured in such a way that it contains information regarding the definition of boundaries and regarding class, may be used directly by geographic information systems.

\section{REFERENCES}

Bauer T., Steinnocher K., 2001, Per-parcel land use classification in urban areas applying a rule-based technique, GIS, vol. 6 .

Brandt T., Mather P.M., 2001, Classification methods for remotely sensed data, Taylor \& Francis.

Corine land cover - Technical guide, 1993.

eCognition User Guide 2001, Definiens Imaging.

de Kok R., Buck A., Schneider T., Ammer U., 2002, Modular project design in object oriented analysis, GIS und Fernerkundung: Neue Sensoren - Innovative Methoden, Wichmann Verlag, Heidelberg: 33-42.

English translation: Joanna M. Kwiatowska 